\title{
El uso de la harina de morro como ingrediente proteínico para la alimentación de tilapia
}

Edgar Osiris Carranza Espinal ${ }^{1}$

\section{RESUMEN}

La tilapia es un cultivo de fácil manejo, sin embargo, el $60 \%$ de los costos totales de producción es la alimentación y dado que las dietas balanceadas se mantienen al alza debido al incremento de algunos insumos como las harinas de pescado, soya y maíz, los acuicultores buscan fuentes alternativas de proteína que abarate los costos de alimentación; ante esta situación, la harina de morro puede ser una opción. El objetivo de este estudio fue evaluar el efecto de la harina de morro como ingrediente proteínico en la alimentación de la tilapia a través del desempeño productivo durante 256 días de cultivo en una dieta experimental, para que contribuya a la reducción de costos de producción para los acuicultores nacionales. El estudio se realizó en el Centro de Investigación Acuícola Pesquero, se usaron cuatro tanques de concreto con $1.86 \mathrm{~m} 3$ de capacidad, en cada tanque se sembraron 45 tilapias (24.2 peces por $\mathrm{m} 3$ ) a un peso inicial de $5 \mathrm{~g}$ y se cosecharon 256 días después. Los peces fueron alimentados con una dieta balanceada al $28 \%$ de PC y una dieta experimental a base de sorgo, melaza y morro (82.7 \%), con $28 \%$ de PC. Se evaluaron indicadores de desempeño y relación biométrica. Los peces alimentados con la dieta balanceada mostraron mejores resultados en los indicadores de desempeño; en los peces alimentados con la dieta experimental, la ganancia diaria de peso fue menor $(P>0.0356)$. Se encontraron los peces con mayor longitud y peso a los alimentados con la dieta comercial; en los dos tratamientos se observó una alta correlación entre longitud y peso $(r>0.90)$. Se sugiere usar la a harina de morro como ingrediente de proteína con la inclusión de alimentos ricos en los aminoácidos de lisina, metionina y treonina.

Palabras clave: dieta balanceada, fuente proteínica, harina de morro, indicadores de desempeño, aminoácidos.

\footnotetext{
${ }^{1}$ Profesor investigador del Centro Regional Universitario de Litoral Pacífico CURLP, UNAH: edgar.carranza@unah.edu
} 


\section{ASBTRACT}

The cultivation of tilapia generated in Honduras 70 million dollars in 2014, positioning the country as the first exporter of fresh fillet of tilapia in the world. Tilapia is an easy-to-handle crop; however, $60 \%$ of the total production costs are feed and since balanced diets are kept on the rise due to the increase in some inputs such as fishmeal, soybeans and maize, Aquaculturists are looking for alternative sources of protein that will lower food costs; In view of this situation, the flour of morro can be an option. The study was carried out at the Aquaculture Fisheries Research Center, four concrete tanks with $1.86 \mathrm{~m} 3$ of capacity were used, in each tank 45 tilapia (24.2 fish per $\mathrm{m} 3$ ) were planted at an initial weight of $5 \mathrm{~g}$ and harvested 256 days later. The fish were fed a balanced diet at $28 \%$ PC and an experimental diet based on sorghum, molasses and nose (82.7\%), with $28 \%$ PC. Performance indicators and biometric relation were evaluated. Fish fed the balanced diet showed better results in performance indicators; In the fish fed the experimental diet, the daily weight gain was lower $(P>0.0356)$. Fish with greater length and weight were found in those fed the commercial diet; In both treatments a high correlation between length and weight was observed $(r>0.90)$. Morro meal can be used as a protein ingredient with the inclusion of foods rich in the amino acids of lysine, methionine and threonine.

Key words: balanced diet, protein source, morro meal, performance indicators, amino acids. 


\section{INTRODUCCIÓN}

El cultivo de la tilapia representa para Honduras una importante actividad económica, así en el año 2014 las exportaciones de filete fresco alcanzaron los 21.6 millones de libras, que equivalen a 70 millones de dólares americanos en divisas, posicionando al país como el mayor exportador de filete fresco de tilapia hacia los Estados Unidos (Espinoza, 2014). Esta actividad genera alrededor de 1,800 empleos directos y 6,000 indirectos $(\mathrm{ClClH}, 2009)$. El cultivo de la tilapia está en crecimiento debido a la demanda del mercado internacional.

Para los piscicultores una de las motivaciones para aumentar el área de cultivo y el volumen de producción de la tilapia, son las características favorables que tiene, tales como: rápido crecimiento, resistencia a enfermedades, fácil reproducción en cautiverio y tolerancia a un gran rango de condiciones ambientales adversas (Cifuentes y Meyer, 2007). Estas características hacen que la tilapia sea la especie acuícola de preferencia por los proyectos de desarrollo rural en sistemas de pequeña y mediana escala de producción.

El éxito económico de la crianza de tilapia depende en gran parte de la alimentación, con el uso de alimentos balanceados comerciales, pero representa el $60 \%$ de los costos totales de producción (Pantoja, Sánchez y Hoyos, 2011). Para los piscicultores usar alimentos balanceados en forma de comprimidos flotantes tiene ventajas como un mayor aprovechamiento del alimento, debido a que sus ingredientes están parcial o totalmente cocidos, facilitando de esta forma la digestión y absorción de nutrientes (Padilla y Meyer, 2004). Además tienen muy buena palatabilidad y atractabilidad, factores que hacen que los peces coman hasta quedar satisfechos.

Sin embargo, los alimentos balanceados para peces se elaboran a base de harina de pescado como fuente principal de proteína, insumo de elevado costo y alta variación de precio en el mercado internacional que tiende al alza (Pantoja, Sánchez y Hoyos, 2011). El precio del alimento balanceado es influenciado por el contenido de proteína cruda y al incrementar su valor se reduce la rentabilidad del cultivo, sobre todo en aquellos productores que no cuentan con capital para comprarlo.

La tilapia tiene hábitos alimenticios muy amplios, puede ser alimentada de manera artificial con subproductos de la agricultura (estiércol de bovinos, porcinos y aves), hojas (yuca), vegetales (papa, repollo), granos básicos (maíz y soya) y alimentos concentrados (Cifuentes y Meyer, 2007). El alza de los precios de los insumos de los 
alimentos balanceados como el maíz, harina de pescado y la soya, el difícil manejo del estiércol (contaminante del agua), la falta de conocimiento y disponibilidad de otros subproductos, son factores que impulsan al productor a encontrar nuevas fuentes complementarias de alimento que estén disponibles localmente (Valle, 2009). El uso de estos productos como alimentos para la tilapia permite reducir costos y tiempo para la cosecha, sobre todo en sistemas extensivos o de subsistencia.

Una alternativa para la sustitución de proteína de harina de pescado es el "morro", semilla extraída del fruto del jícaro (Crescentia alata y Crescentia cujete). El árbol es una especie vegetal de la familia Bignoniaceae, característico del bosque seco y nativo de la región mesoamericana (Galeano, 2011); se produce de forma silvestre y llega a tener una altura de ocho metros, es tolerante a la sequía y las altas temperaturas, produce frutos en forma de calabaza esférica con una cáscara dura y leñosa y una pulpa que contiene de 300 a 900 semillas, lo que es aprovechado para la alimentación del ganado bovino durante el pastoreo (Flores, 2010). La semilla seca por cada $100 \mathrm{~g}$ contiene $30.2 \mathrm{~g}$ de proteína, $39.7 \mathrm{~g}$ de grasas, $22.9 \mathrm{~g}$ de carbohidratos totales, $2.4 \mathrm{~g}$ de fibra, $3.4 \mathrm{~g}$ de agua y aporta 5,300 Kcal/kg (Zuñiga, 2007). Todas las anteriores son características nutricionales favorables que pueden aprovecharse para la alimentación de la tilapia.

El objetivo de este estudio fue evaluar el efecto de la harina de morro como ingrediente proteínico en la alimentación de la tilapia a través del desempeño productivo durante 256 días de cultivo en una dieta experimental, para que contribuya a la reducción de costos de producción para los acuicultores nacionales. Y de esta manera comparar la ganancia de peso de las tilapias alimentadas en períodos alternos con una dieta balanceada comercial y una dieta experimental a base de harina de morro. Así como evaluar los indicadores de desempeño durante tres fases de crecimiento del cultivo. $Y$ relacionar la longitud y el peso de acuerdo a la ecuación de crecimiento.

\section{MATERIALES Y MÉTODO}

El estudio se realizó durante los meses de marzo a diciembre de 2014, en el Centro de Investigación Acuícola Pesquero, ubicado en la ciudad de San Lorenzo, departamento de Valle, a una altura de $4 \mathrm{msnm}$ y con una temperatura promedio anual de $34{ }^{\circ} \mathrm{C}$. 


\section{Diseño experimental}

Se utilizaron 180 alevinos obtenidos de una granja comercial de producción y fueron alojados en cuatro tanques cuadrado de concreto de $1.859 \mathrm{~m} 3(1.9 \mathrm{~m} \times 0.87 \mathrm{~m} \mathrm{x}$ $1.125 \mathrm{~m}$ ). La densidad inicial por tanque fue de 45 peces ( 24.2 peces por $\mathrm{m} 3$ ) y disminuyó en función del tiempo debido a la mortalidad. Los peces a la siembra tenían $5 \mathrm{~g}$ de peso y $4.5 \mathrm{~cm}$ de longitud. Diariamente fue registrada la temperatura y la turbidez, realizando los manejos adecuados para lograr las óptimas condiciones de calidad del agua para el desarrollo de los peces.

Se establecieron tres fases de crecimiento (crecimiento 1, crecimiento 2 y finalización) en todo el experimento, cada fase comprendió diferentes días de cultivo, que dependió del tiempo de los peces para lograr un peso determinado. De los cuatro tanques, en dos se usó el alimento experimental y en los otros dos se alimentó con pienso balanceado para tilapia al $28 \%$ PC. En los tanques en donde se probó la dieta experimental, en las tres fases de crecimiento se alimentó los primeros 65 días con la dieta experimental y los siguientes días con el alimento balanceado comercial al $28 \%$ de proteína cruda (PC), hasta completar la siguiente fase de crecimiento.

La dieta experimental consistió en la mezcla homogénea de $10.3 \%$ de harina de sorgo, $82.7 \%$ de harina de morro y $7 \%$ de melaza de caña; a la mezcla se le adicionaron $600 \mathrm{ml}$ de agua a $90{ }^{\circ} \mathrm{C}$ de temperatura y se 'introdujo en un molino de carne para formar los pellets. Seguidamente recibieron un tratamiento térmico en un horno a $150{ }^{\circ} \mathrm{C}$ durante 45 minutos y radiación solar por 10 horas. El agua caliente contribuye a la cocción de los almidones del sorgo y el morro para optimizar las propiedades aglutinantes de la mezcla; la cocción en el horno previene la formación de hongos en el alimento y contribuye a la flotabilidad del pellet; el secado solar ayuda a reducir el contenido de humedad en el pellet (Valencia, Montejo y Carrillo, 2014). Los pellets secos se ajustaron al tamaño del pez según su fase de crecimiento; en la primera fase de crecimiento fueron más pequeños y se aumentaron en las siguientes fases de crecimiento. Con esta mezcla se logró una dieta con un $26.2 \%$ de proteína cruda.

La cantidad diaria del alimento fue calculada como porcentaje de la biomasa de los peces en cada tanque (ver cuadro 1) y fue dividida en dos porciones diarias (8:00 am. y 4:00 pm.). La ración de alimento aplicada fue cambiaba después de la realización de cada muestreo de crecimiento. 


\section{Cuadro 1. Alimentación para tilapias según la recomendación del fabricante}

\begin{tabular}{|c|c|}
\hline Peso promedio del pez en gramos & Porcentaje del alimento según el peso vivo del pez \\
\hline 5 & 9.50 \\
10 & 7.00 \\
25 & 5.00 \\
50 & 3.25 \\
100 & 2.90 \\
120 & 2.50 \\
150 & 2.00 \\
200 & 1.70 \\
\hline
\end{tabular}

Fuente propia

En el análisis proximal y la densidad calórica en términos de energía digestible según la Association of Official Analytical Chemistsy (2005), los valores fueron determinados por el fabricante en la dieta balanceada comercial y en la dieta experimental fueron estimados por tablas nutricionales de ingredientes para balanceados (cuadro 2). El análisis proximal presenta la composición nutricional de los alimentos en cuanto a proteína cruda, humedad, fibra, cenizas, calcio y fósforo (Olvera, Palacios y León, 1993).

\section{Cuadro 2. Composición proximal de las dietas usadasdas en el sistema de} alimentación

\begin{tabular}{|l|l|l|}
\hline Componente & Dieta experimental (\%) & Dieta balanceada (\%) \\
\hline Humedad & 6.0 & 13.0 \\
Proteína cruda & 26.2 & 28.0 \\
Fibra cruda & 2.2 & 5.0 \\
Cenizas & 4.0 & 7.0 \\
Calcio & 0.1 & 1.0 \\
Fósforo & 0.8 & 0.8 \\
Energía bruta Kcal/Kg & $4,843.0$ & $3,740.0$ \\
\hline
\end{tabular}

Fuente: Cargill, 2015, modificada por el autor

Todos los animales recibieron las mismas condiciones de manejo, se hicieron recambios parciales del $40 \%$ de agua de cada tanque todos los días y cada ocho días se realizaba un recambio total del agua. No se contó con sistema de aireación, razón por la que se realizaron los recambios de agua constantemente. 


\section{Intervenciones}

Semanalmente se realizaron pesos y mediciones de los peces, estos fueron capturados manualmente con una red de mano. Para cada una de las fases se utilizaron los siguientes indicadores de desempeño:

1. Ganancia diaria $(\mathrm{g} / \mathrm{pez})=(\mathrm{Pf}-\mathrm{Pi}) / \mathrm{tac}$

2. Factor de conversión alimenticia $=\mathrm{TAS} / \mathrm{BTP}$

3. Factor de condición $=100^{*}(\mathrm{P} / \mathrm{Lt} 3)$

4. Tasa específica de crecimiento $(\% /$ día $)=100^{*}(\mathrm{LnPf}-\mathrm{LnPi}) / \mathrm{t}$

5. Crecimiento relativo $(\%)=(\mathrm{Pf}-\mathrm{Pi}) / \mathrm{Pi})^{*} 100$

6. Supervivencia $(\%)=100^{*}(\mathrm{Pv} / \mathrm{Psi})$

Dónde:

$\mathrm{Pf}=$ peso promedio final de cada fase de crecimiento.

$\mathrm{Pi}=$ peso promedio inicial de cada fase de crecimiento.

Tac= número de días acumulados hasta el final de cada una de las fases.

TAS = total de alimento suministrado por fase de crecimiento.

BTP = biomasa total de los peces en cada fase de crecimiento.

$\mathrm{P}=$ peso corporal de los peces al final de cada fase de crecimiento.

$\mathrm{t}=$ tiempo de duración de las fases de crecimiento en días.

$\mathrm{Lt}=$ longitud total de cada uno de los peces muestreados al final de cada fase.

LnPf= logaritmo natural del peso corporal promedio final de cada una de las fases de crecimiento.

$\mathrm{LnPi}=$ logaritmo natural del peso corporal promedio inicial de cada una de las fases de crecimiento.

$P v=$ peces vivos al final de cada fase de crecimiento.

$P s i=$ peces sembrados inicialmente.

\section{Análisis estadístico}

En las comparaciones de ganancia de peso de los peces se usó la comparación de medias de la prueba t al nivel de significancia del 0.05 e indicadores de desempeño, se utilizó estadística descriptiva calculando media aritmética y desviaciones estándar y la prueba t student para las comparaciones de las medias; en la relación biométrica se usó el análisis de relación. Los datos fueron analizados con el programa estadístico InfoStat versión 2008. 


\section{RESULTADOS}

\section{Crecimiento de los peces}

Los peces presentaron pesos similares hasta los 113 días de cultivo, a medida que crecieron los animales se observó una mejor ganancia de peso en las tilapias alimentadas con el alimento comercial. La diferencia de los pesos finales fue de 15 gramos más para las tilapias alimentadas con la dieta comercial (cuadro 3); estadísticamente se observaron resultados concluyentes $(P>0.0356)$ para los pesos y longitudes finales de los peces en donde el alimento comercial mostró los mejores resultados.

Cuadro 3. Peso corporal y longitud obtenido en cada uno de las fases de alimentación de los peces.

\begin{tabular}{|l|c|c|c|c|c|c|}
\hline \multicolumn{2}{|l|}{} & \multicolumn{3}{|c|}{ Pesos final obtenido $(\mathrm{g})$} & \multicolumn{2}{l|}{$\begin{array}{l}\text { Longitud final obtenida } \\
(\mathrm{cm})\end{array}$} \\
\hline $\begin{array}{l}\text { Fase de } \\
\text { crecimiento }\end{array}$ & $\begin{array}{l}\text { Duración } \\
\text { en días }\end{array}$ & $\begin{array}{l}\text { Días } \\
\text { acumulados }\end{array}$ & $\begin{array}{l}\text { Alimento } \\
\text { comercial }\end{array}$ & $\begin{array}{l}\text { Dieta } \\
\text { experimental }\end{array}$ & $\begin{array}{l}\text { Alimento } \\
\text { comercial }\end{array}$ & $\begin{array}{l}\text { Dieta } \\
\text { experimental }\end{array}$ \\
\hline Crecimiento 1 & 78 & 78 & 40.8 & 36.6 & 9.4 & 9.9 \\
Crecimiento 2 & 106 & 184 & 107.3 & 92.6 & 12.0 & 11.9 \\
Finalización & 70 & 254 & 147.7 & 132.7 & 20.8 & 19.9 \\
\hline
\end{tabular}

Fuente propia

\section{Indicadores de desempeño}

La mejor ganancia diaria de peso $(\mathrm{g} / \mathrm{m} 3)$ se observó en los peces que consumieron alimento comercial en las primeras dos fases de crecimiento; en la fase de finalización la ganancia diaria de peso fue igual en los dos tratamientos de alimento. En el factor de conversión alimenticia se encontró una mayor eficiencia de aprovechamiento del alimento en la etapa de crecimiento 2, para los dos tipos de dietas; en las etapas de crecimiento 1 y finalización, se usó más de un kilogramo de alimento para lograr un kilogramo de peso de los peces (cuadro 4). 


\section{Cuadro 4. Índice de desempeño productivo usando dos dietas en el cultivo de la} tilapia evaluados en tres fases de crecimiento

\begin{tabular}{|c|l|c|c|c|c|c|c|}
\hline $\begin{array}{l}\text { Fase de } \\
\text { crecimiento }\end{array}$ & $\begin{array}{l}\text { Tipo de } \\
\text { alimento }\end{array}$ & $\begin{array}{l}\text { Ganancia } \\
\text { diaria } \\
\left(\mathrm{g} / \mathrm{m}^{3}\right)\end{array}$ & $\begin{array}{l}\text { Factor de } \\
\text { conversión } \\
\text { alimenticia }\end{array}$ & $\begin{array}{l}\text { Factor de } \\
\text { condición }\end{array}$ & $\begin{array}{l}\text { Tasa } \\
\text { específica de } \\
\text { crecimiento } \\
\text { (\%/dia) }\end{array}$ & $\begin{array}{l}\text { Crecimiento } \\
\text { relativo (\%) }\end{array}$ & $\begin{array}{l}\text { \% de } \\
\text { especie }\end{array}$ \\
\hline $\begin{array}{c}\text { Crecimiento } \\
1\end{array}$ & $\begin{array}{l}\text { Alimento } \\
\text { comercial }\end{array}$ & 0.46 & 2.60 & 4.99 & 2.69 & 715.60 & 100.00 \\
\hline & $\begin{array}{l}\text { Alimento } \\
\text { alternativo }\end{array}$ & 0.41 & 2.87 & 3.83 & 2.55 & 632.00 & 100.00 \\
\hline $\begin{array}{c}\text { Crecimiento } \\
2\end{array}$ & $\begin{array}{l}\text { Alimento } \\
\text { comercial }\end{array}$ & 0.60 & 1.60 & 5.73 & 0.84 & 142.70 & 71.10 \\
\hline & $\begin{array}{l}\text { Alimento } \\
\text { alternativo }\end{array}$ & 0.42 & 1.58 & 5.53 & 0.62 & 92.10 & 64.70 \\
\hline Finalización & $\begin{array}{l}\text { Alimento } \\
\text { comercial }\end{array}$ & 0.52 & 2.34 & 1.65 & 0.41 & 33.10 & 71.10 \\
\hline & $\begin{array}{l}\text { Alimento } \\
\text { alternativo }\end{array}$ & 0.52 & 2.35 & 1.68 & 0.45 & 37.40 & 63.60 \\
\hline
\end{tabular}

Fuente propia

Con relación al factor de condición, se observaron los mayores valores en la fase de crecimiento 2 para los dos tratamientos; en la fase de finalización estos valores se reducen. En la tasa específica de crecimiento, los peces lograron un mayor crecimiento en la primera fase ( 2.69 y $2.55 \%$ ), a medida que crecieron ganaban peso; el porcentaje de crecimiento diario disminuyó, observándose un mejor desempeño en los peces alimentados con la dieta comercial. En el crecimiento relativo los peces alimentados con la dieta comercial presentaron un mayor desarrollo en las fases $1 \mathrm{y}$ 2; en la etapa de finalización los peces alimentados con el alimento artesanal mostraron un mejor desempeño.

En la supervivencia, los peces alimentados con la dieta balanceada presentaron un mayor número de peces vivos durante la cosecha, sin embargo, estos valores estadísticamente no fueron concluyentes $(P<0.0895)$, ya que las mortalidades se presentaron por causa del manejo y no por efecto del alimento.

\section{Relaciones biométricas}

Se encontraron los peces con mayor longitud y peso en los alimentados con la dieta 
comercial, el coeficiente de regresión se ajustó al modelo potencial para poder predecir el peso de la tilapia en función de la longitud (peso total $=0.3941 \mathrm{x}^{2.0586}$ ), siendo $\mathrm{x}$ la longitud del pez. Se observó una alta correlación entre las dos variables (ver figura 1), el coeficiente de regresión fue altamente significativo $\left(R^{2}=0.889\right)$, demostrando una alta relación entre las variables estudiadas.

Los peces alimentados con la dieta alternativa mostraron un progresivo crecimiento durante el estudio, la longitud obtenida en cada fase de crecimiento fue parecida a la de los peces con tratamiento de la dieta balanceada, pero los pesos fueron menores. En el análisis biométrico el modelo potencial es ajustado a la predicción de los pesos totales en función de la longitud del pez (peso total $=0.4023 \mathrm{x}^{2.0428}$ ), muy parecido a ecuación anterior; se presentó una alta relación entre variables (ver figura 2) con un coeficiente de determinación mayor del $88 \%\left(R^{2}=0.905\right)$, demostrando en ambos casos una alta confiabilidad estadística para la predicción de los pesos totales de los peces.

Figura 1. Relación longitud y peso de tilapias alimentadas con dieta balanceada comercial en el Centro de Investigación Acuícola Pesquero en el año 2014

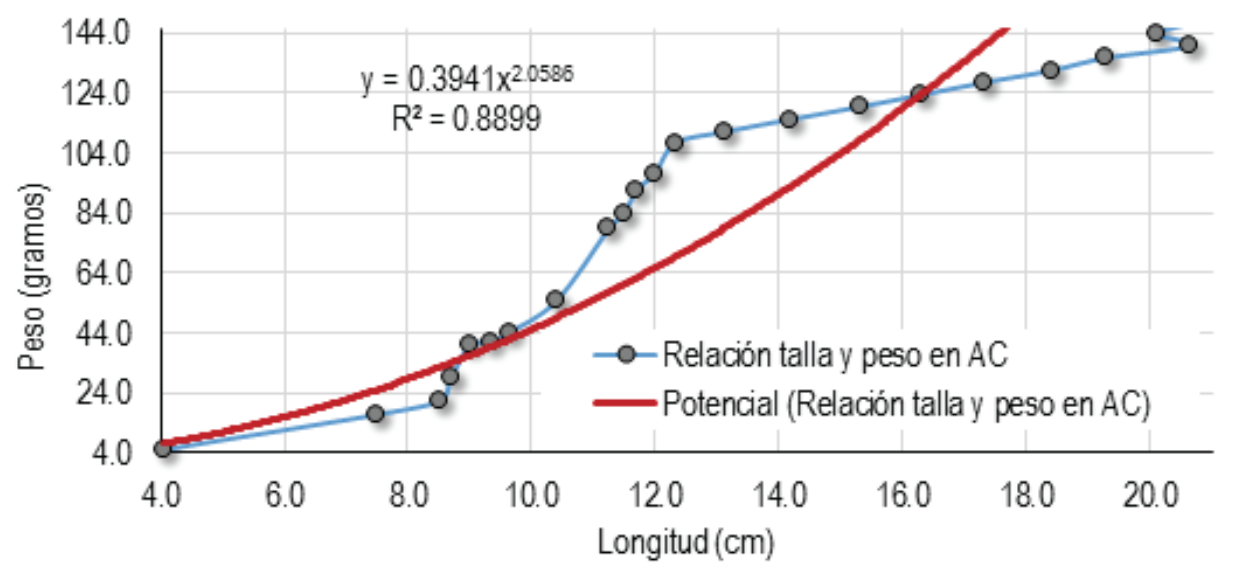

Fuente propia 
Figura 2. Relación longitud y peso de tilapias alimentadas con dieta experimental (inclusión de harina de morro) en el Centro de Investigación Acuícola Pesquero en el año 2014.

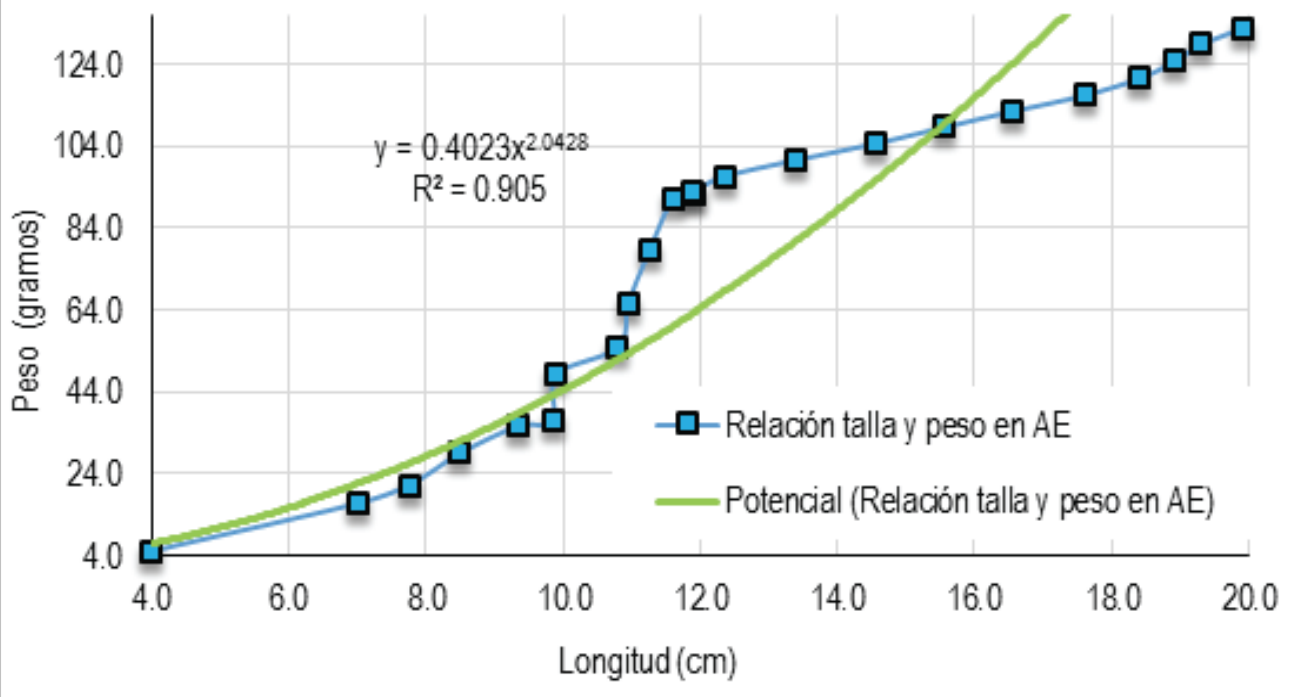

Fuente propia

\section{DISCUSIONES}

Las tilapias en general presentaron pesos finales por debajo del peso promedio de un cultivo, pues un pez manejado en óptimas condiciones tiene una ganancia diaria de peso de más de $1.20 \mathrm{~g}$ (Meyer, 2004). Los peces en el ensayo presentaron ganancias diarias entre 0.4 y $0.6 \mathrm{~g}$, esto se debió a la ausencia de un sistema aireación externa, ya que el oxígeno disuelto disponible para el cultivo dependía de las reservas que tenía la columna de agua de los tanques, siendo muchas veces insuficiente durante la noche. Los peces al sufrir deficiencias de oxígeno aletargan su metabolismo y entran en estado de supervivencia; su capacidad de alimentarse es restringida (Mallya, 2012) cuando se presentan eventos de deficiencia de oxígeno disuelto (menores de 3 partes por millón) y los efectos en el pez duran hasta siete días (Meyer, 2004).

A pesar de que los peces mostraron bajo crecimiento con relación al tiempo de cultivo, el factor de condición como indicador de desempeño fue muy favorable en 
todas las fases de crecimiento, sobre todo en la fase crecimiento 2; las diferencias de este indicador entre los tratamientos no fueron concluyentes $(P>0.0878)$. El factor de condición define los cambios de condición de los peces en cuanto a la edad, sexo y ciclo estacional, también mide la efectividad de los programas de alimentación en la acuicultura (Fonticiella, 2009). El índice de condición es la gordura relativa de los peces.

Las diferencias encontradas en los pesos y longitudes de los peces alimentados con la dieta experimental se debe a que la harina de morro es deficiente en los aminoácidos esenciales de lisina, metionina y treonina, que son los requerimientos para tilapia recomendados por Torres y Hurtado (2012), quienes sugieren que los niveles de lisina, metionina y treonina deben ser de $1.38 \%, 0.47 \%$ y $1.07 \%$, respectivamente. El aporte estimado de los aminoácidos esenciales de la dieta experimental fue de $0.44 \%$ de lisina, $0.08 \%$ de metionina y $0.49 \%$ de treonina. Las deficiencias de estos aminoácidos evidencian en los peces menor apetito, menor ingesta de alimentos y la presencia de deficiencias nutricionales presentes como lordosis y escoliosis (Castro y Avilia, 1994). Los peces que usaron la dieta experimental no presentaron estas deficiencias debido a los períodos que se alimentaron con la dieta balanceada entre las fases de crecimiento, pero sí influyó la deficiencia de estos aminoácidos en la ganancia de peso.

Con relación a la biométrica de los peces, se encontraron valores muy parecidos en ambos casos, las correlaciones y las regresiones demostraron que los pesos y las longitudes están muy relacionados directa y proporcionalmente; las ecuaciones obtenidas son confiables, por tanto, pueden usarse para predecir pesos de las tilapias en función de la longitud del pez.

Las supervivencias estuvieron entre el $70 \%$ y $63 \%$ de la cosecha en los dos tratamientos, muchos peces durante el experimento murieron por la baja saturación de oxígeno disuelto durante la noche, debido al proceso fotosintético de las microalgas que durante el día liberan oxígeno como producto secundario de la reacción bioquímica a la fotosíntesis, saturando hasta con un $200 \%$ de oxígeno disuelto en horas de la tarde (Fraga, Flores, Reyes y Llanes, 2012). Por la noche no hay liberación de oxígeno y todos los organismos, incluyendo las microalgas, respiran, reduciendo la cantidad de oxígeno disuelto hasta niveles letales para las tilapias. 


\section{CONCLUSIONES}

La harina de morro puede usarse como fuente proteínica principal en una dieta alternativa para alimentar tilapias, pero fortalecida con la inclusión de otros ingredientes ricos en lisina, metionina y treonina, como la torta de maní o torta de ajonjolí, productos que se encuentran en la región a bajo costo.

Los peces que lograron los mejores indicadores de desempeño fueron los alimentados con la dieta balanceada, ya que el aporte de aminoácidos cubría los requerimientos nutricionales de la tilapia; la dieta experimental presentó deficiencias en los aminoácidos de lisina, metionina y treonina, aletargando el crecimiento de los peces. La dependencia en la producción natural de oxígeno contribuyó a que los peces no desarrollaran un crecimiento óptimo durante el desarrollo del experimento, afectando su proceso natural de crecimiento.

\section{BIBLIOGRAFÍA}

Association of Official Analytical Chemists. (2005). Official Methods of analysis. EE.UU.: AOAC.

Castro, E. y Avilia, L. (1994). Control de calidad de insumos y dietas acuícolas. México. FAO. Recuperado de: http://www.fao.org/docrep/field/003/ab482s/ ab482s06.htm

$\mathrm{CIClH}$. (2009). Oportunidades de inversión sector agronegocios. Camara de Industria y Comercio Italo-Hondureña. Recuperado de: http://www.cicih.com/assets/files/proyectos

Cifuentes, G. J. y Meyer, D. (2007). Alimentación de la tilapia del Nilo (Oreochromis niloticus) con harina del fruto de guanacaste (Enterolobium ciclocarpum). Recuperado de: http://bdigital.zamorano.edu/handle/11036/843

Espinoza, J. (18 de febrero de 2014). Honduras, primer exportador de filete fresco de tilapia a Estados Unidos. Diario La Prensa. Recuperado de: http://www.laprensa.hn/economia/laeconomia/476355-98/honduras-primer-exportador-de-filete -fresco-de-tilapia-a-eua

Flores, E. J. (2010). Evaluación de la pulpa ensilada del totumo (Crescentia cujete I) en dos estados de maduración (totumo verde y totumo amarillo) como alternativa en la alimentación bovina. Universidad del Zulia, Maracaibo. Recuperado de: http://tesis.luz.edu.ve/tde_busca 
Fonticiella, D. W. (2009). Peso estándar de la tilapia (Oreochromis aureus Steindachner) en Cuba. Revista de producción animal, 12, 5-12. Recuperado de: http://www.reduc.edu.cu/147/00/14700101.pdf

Fraga, I.; Flores, E.; Reyes, R. y Llanes, Y. (2012). Efecto de diferentes densidades de siembra en el engorde de tilapia roja (Oreochromis mossambicus $\times 0$. aureus) en jaulas colocadas en la bahía de Casilda, Cuba. Revista de investigaciones marinas, 32(1), 16-23. Recuperado de: http://www.cim.uh.cu/rim/pdf/2012/1/ 16-23.pdf

Galeano, M. M. (2011). Explotación sistemática del totumo (Crescentia cujete L y Crescentia alata K) en silvopastoreo, producción de forraje, frutos para alimentación animal y farmacopea. Alternativa sostenible para afrontar el cambio climático. Recuperado de: http://www.engormix.com/MA-ganaderia-carne/articulos/explotacion-sistematica-totumo-crescentia-t3459/p0.htm

Mallya, Y. J. (2012). Los efectos del oxígeno disuelto en el crecimiento de los peces en la acuicultura. Aqua Feed International. Recuperado de: http://aquafeed.co/los-efectos-del-oxigeno-disuelto-en-el-crecimiento-de-los-peces-en-laacuicultural

Meyer, D. (2004). Introducción a la acuacultura. Honduras: Escuela Agrícola Panamericana.

Olvera, M. Á.; Palacios, C. M. y León, E. R. (1993). Manual de técnicas para laboratorio de nutrición de peces y crustáceos. FAO. Recuperado de: http://www.fao.org/docrep/field/003/ab489s/ab489s03.htm

Padilla, K. N. y Meyer, D. (2004). Evaluación de cuatro formas físicas de alimento concentrado para engorde de tilapia. Recuperado de: http://bdigital.zamorano.edu /bitstream/11036/2126/1/T1968.pdf

Pantoja, J. O.; Sánchez, S. M. y Hoyos, J. L. (2011). Obtención de un alimento extraído para tilapia roja (Oreochromis spp) utilizando ensilaje biológico de pescado. Biotecnología en el sector Agropecuario y Agroindustrial, 9(2), 178-187. Recuperado de: http://www.scielo.org.co/scielo.php

Purina. (2015). Tablas de alimentación para tilapia. Recuperado de: http://www.nutrimentospurina.com.pe/Screens/Tilapia1.aspx

Torres, D. M. y Hurtado, V. (2012). Requerimientos nutricionales para tilapia del Nilo. Orinoquia, 16(1), 63-68. Recuperado de: http://orinoquia.unillanos.edu.co/index.php/orinoquia/article/view/266

Valencia, J. H.; Montejo, G. y Carrillo, L. (2014). Manual elaboración de alimento alternativo para la producción de tilapia. Recuperado de: http://siproduce.sifupro.org.mx/seguimiento/archivero/20/2013/anuales/anu_706-25-2014-05-7.pdf 
Valle, E. L. (2009). Evaluación productiva de tres protocolos para alimentar tilapia del Nilo. Recuperado de: http://bdigital.zamorano.edu/bitstream/11036/2008/1/T16 59.pdf

Zuñiga, G. R. (2007). Análisis físico químico y evaluación del rendimiento de extracción del aceite de semilla de morro (Crescentia alata) proveniente de las regiones de Estanzuela, Zacapa y San Agustín Acasaguastlán, El Progreso. Universidad de San Carlos de Guatemala. Recuperado de: http://biblioteca.usac.edu.gt/tesis/08/08_1036_Q.pdf 